\title{
Review
}

\section{Deconstructing the urinalysis: A novel approach to diagnostic and antimicrobial stewardship}

\author{
Sonali D. Advani MBBS, MPH ${ }^{1,2}$ (1), Christopher R. Polage MD, MAS ${ }^{3}$ and Mohamad G. Fakih MD, MPH ${ }^{4,5}$ \\ ${ }^{1}$ Division of Infectious Diseases, Duke University School of Medicine, Durham, North Carolina, ${ }^{2}$ Duke Infection Control Outreach Network, Durham, North \\ Carolina, ${ }^{3}$ Department of Pathology, Duke University School of Medicine, Durham, North Carolina, ${ }^{4}$ Quality Department, Clinical \& Network Services, Ascension \\ Health, St. Louis, Missouri and ${ }^{5}$ Wayne State University School of Medicine, Detroit, Michigan
}

\begin{abstract}
The extensive use of the urinalysis for screening and monitoring in diverse clinical settings usually identifies abnormal urinalysis parameters in patients with no suspicion of urinary tract infection, which in turn triggers urine cultures, inappropriate antimicrobial use, and associated harms like Clostridioides difficile infection. We highlight how urinalysis is misused, and suggest deconstructing it to better align with evolving patterns of clinical use and the differential diagnosis being targeted. Reclassifying the urinalysis components into infectious and non-infectious panels and interpreting urinalysis results in the context of individual patient's pretest probability of disease is a novel approach to promote proper urine testing and antimicrobial stewardship, and achieve better outcomes.
\end{abstract}

(Received 30 April 2021; accepted 7 May 2021)

A perceived "abnormal" urinalysis result usually leads to the initiation of antimicrobials, often regardless of genitourinary symptoms. ${ }^{1}$ Early in training, most medical students and residents learn to view pyuria, bacteriuria, and infection interchangeably.,3 Many clinicians order urine cultures and prescribe antibiotics inappropriately in asymptomatic patients with abnormal urinalysis parameters, often regardless of genitourinary symptoms ${ }^{1}$ contrary to national guidance. $^{4,5}$ Recently, many US hospitals and medical centers have focused on reducing inappropriate urine cultures and leveraging the use of the urinalysis prior to obtaining a culture as diagnostic stewardship interventions. ${ }^{6-9}$ Such interventions have been influenced by biased economic incentives linked to catheter-associated urinary tract infection (CAUTI) prevention. ${ }^{10}$ Even though diagnostic stewardship interventions generally result in a reduction in the number of urine cultures ordered, their impact on appropriate antimicrobial use or clinician's response to an abnormal urinalysis is not clear.

\section{Utility of urinalysis}

The urinalysis is a popular screening test used across a wide range of inpatient and outpatient clinical settings, due to ease of accessibility, rapidity of results, and low cost. It is useful in the diagnosis and progression of wide range of medical conditions such as renal calculi, metabolic disorders, diabetes, acute and chronic kidney diseases, infections, stroke, and malignancy. ${ }^{11,12}$ Urinalysis evolved over

Author for correspondence: Sonali Advani, MBBS, MPH, Duke Infection Control Outreach Network (DICON), Division of Infectious Diseases, Duke University Medical Center, 315 Trent Drive, Hanes House, Room 154, Durham, NC 27710. E-mail: sonali. advani@duke.edu

Cite this article: Advani SD, Polage CR, and Fakih MG. (2021). Deconstructing the urinalysis: A novel approach to diagnostic and antimicrobial stewardship. Antimicrobial Stewardship \& Healthcare Epidemiology, https://doi.org/10.1017/ash.2021.167 the last 200 years to include different chemical analyses and microscopic examination, ${ }^{13}$ making it a compilation of unaligned tests that only have their specimen source in common. ${ }^{14}$ Contrary to blood tests, where metabolic and hematologic panels are separately ordered, urinalysis does not have separate panels for workup of infection, metabolic disorders, or renal disease. As a result, physicians order a complete urinalysis for wide variety of reasons from general screening to cancer detection. Likewise, urinalysis is also overused to diagnose urinary tract infections in patients with nonspecific symptoms like confusion, fever, abdominal pain or sepsis without genitourinary symptoms. This has led to overuse of urinalysis in different settings, with $60 \%-80 \%$ of urinalyses being ordered in patients without symptoms referable to the genitourinary tract. ${ }^{15-17}$

\section{Urinalysis components}

The different components of urinalysis, as it is done today, are described below:

(1) Gross examination of urine includes description of color, odor, clarity, volume, and specific gravity. Urine color, clarity and volume may be altered due to many etiologies like dehydration, diet, medications, liver disease, infections, hematuria, and certain medical conditions.

(2) Chemical examination of urine reflects parameters that may be encountered in a variety of acute and chronic illnesses. The urine dipstick is a rapid semiquantitative assessment of parameters such as $\mathrm{pH}$, heme, albumin, specific gravity, glucose, leukocyte esterase, and nitrite.

Leukocyte esterase and nitrite have been traditionally used to evaluate for urinary tract infection (UTI). A positive test for

(c) The Author(s), 2021. Published by Cambridge University Press on behalf of The Society for Healthcare Epidemiology of America. This is an Open Access article, distributed under the terms of the Creative Commons Attribution licence (http://creativecommons.org/licenses/by/4.0/), which permits unrestricted re-use, distribution, and reproduction in any medium, provided the original work is properly cited. 
leukocyte esterase may be seen in genitourinary inflammation, irritation from instrumentation or catheterization, glomerulonephritis, UTIs and sexually transmitted infections. ${ }^{3}$ Leukocyte esterase has a good negative predictive value but poor positive predictive value to diagnose infection. ${ }^{18-20}$ A positive test for nitrite can indicate presence of gram-negative bacteriuria, but it does not diagnose UTI in the absence of symptoms. Similarly, a negative test for nitrite does not rule out UTI, as some urinary pathogens like enterococcus do not produce nitrite. In addition, false-positive results for nitrite occurs on exposure to air or phenazopyridine, or from preanalytic contamination. As such, nitrite has poor sensitivity and specificity for diagnosing a UTI. ${ }^{18}$

(3) Microscopic examination of the urine enables confirmation of urine dipstick findings and also the identification of structures that are not evaluated by the urine dipstick (eg, epithelial cells, casts, crystals). It provides further information on inflammatory and non-inflammatory conditions. The presence of white blood cells (WBCs) in urine, also known as pyuria, is indicative of genitourinary inflammation. Pyuria occurs in $32 \%$ of young women, $90 \%$ of elderly patients in long-term care facilities, and $90 \%$ of hemodialysis patients with asymptomatic bacteriuria. ${ }^{1}$ Varying thresholds of pyuria [WBCs $>5 /$ high-powered field (hpf) vs WBCs $>10 / \mathrm{hpf}$ ] do not reliably predict bacteriuria or infection. ${ }^{3,21}$ Even though the absence of pyuria rules out infection, the positive predictive value of pyuria for identifying bacteriuria and UTI is low. ${ }^{17,22}$ Hematuria is also not a reliable predictor of infection. Red blood cells may be present in other medical conditions such as acute glomerulonephritis, stone disease, trauma, malignancy, or menstruation. ${ }^{23,24}$ On the other hand, large numbers of squamous epithelial cells $(>5 / \mathrm{hpf})$ may indicate a poorly collected sample. Renal epithelial cells may indicate renal tubular injury. ${ }^{25,26}$ Casts and crystals in urine may be benign or may represent underlying kidney disease (eg, nephrolithiasis, acute kidney injury) resulting from endogenous crystal production, exogenous drug exposure, inherited diseases, metabolic disorders, and/or drug exposure. ${ }^{12}$

The microscopic examination can also provide information on the presence of microorganisms in the urine but the clinical value has not been systematically studied and likely varies between populations. ${ }^{27}$ Most are performed by automated flow cytometry or image analysis with or without manual microscopic confirmation and cannot distinguish pathogens from nonpathogens or viable from nonviable organisms. Hence, detection of bacteria on the microscopic examination may be associated with positive urine cultures, but it cannot differentiate between asymptomatic bacteriuria, contamination, and UTI. Detection of yeast on the microscopic examination is usually secondary to colonization of urinary tract, indwelling catheter, or vaginal flora. Rarely, it may be due to a true yeast UTI, which is seen in neonates or patients with recent urologic instrumentation or surgery. ${ }^{28}$

\section{Misuse of urinalysis}

Misuse of urinalysis can occur in all stages of testing: pre-analytic, analytic, and post-analytic phases. Misuse during the pre-analytic (ordering) phase occurs when urinalysis is ordered inappropriately for general screening or as a part of a noninfectious disease-specific workup. Urinalysis has been included in many screening and diagnostic protocols in emergency departments, medicine, pediatrics, nursing homes, outpatient clinics, and preoperative assessments. ${ }^{29-32}$ It is often bundled with other screening tests that are not related to an infectious diagnosis. Yin et $\mathrm{al}^{15}$ found that urinalysis was ordered in $62 \%$ of general medicine inpatients, but most of these patients (84\%) were asymptomatic. In a national prevalence study of the urine testing, a urinalysis was ordered in almost half of the admissions. ${ }^{33}$ In another study of patients cared for in the emergency department, more than one-third of urinalyses were done without specific symptoms. ${ }^{17}$ There are also reports of monthly or quarterly standing urinalysis orders placed on nursing-home residents without any specific indications. ${ }^{34} \mathrm{~A}$ similar practice occurs in the ambulatory setting where urinalysis is ordered as an annual screening test (eg, order sets or potentially regular practices in an office for diabetics or psychiatric patients). ${ }^{31,32}$ Likewise, some surgeons order urinalysis or urine culture as a screening test in asymptomatic patients prior to joint replacement procedures based on expert opinion, low-quality evidence, and conflicting evidence. ${ }^{16,30,35}$

In the analytic phase, modifications to laboratory processing of urinalysis to reduce urine cultures may paradoxically lead to the misuse of urinalysis. For example, many US hospitals and laboratories use reflex urine cultures. ${ }^{6,36,37}$ In this approach, when a urinalysis is ordered, it automatically reflexes to urine culture when specific urinalysis parameters (eg, leukocyte esterase, nitrite, white blood cells, yeast or bacteria) are positive alone or in combination. These reflex algorithms became popular in the United States because of the emphasis on CAUTI prevention ${ }^{6,9}$ and the inclusion of CAUTI in the Centers for Medicaid and Medicare Services hospital-acquired-condition reduction program. ${ }^{38}$ Biased economic incentives have led to widespread adoption of reflex urine cultures in many settings. ${ }^{39}$ Although reflex urine cultures have resulted in a reduction in urine culture orders in patients without pyuria, this practice should be avoided in asymptomatic patients or those not suspected to have a UTI. ${ }^{40}$ Additionally, laboratories use different urinalysis parameters and cutoffs to proceed to culture, which leads to confusion and lack of standardized care. ${ }^{36}$ To complicate matters, different forms of urinalysis orderables exist within the same hospital as well as across multiple laboratories without guidance related to pretest probability or underlying diagnosis (eg, urinalysis macroscopic with reflex to microscopic urinalysis, complete urinalysis, urinalysis with reflex to culture, and/or urinalysis dipstick). ${ }^{37}$ Differences in laboratory processing and reporting of urinalyses make comparison of results across different hospitals, ambulatory clinics, and emergency departments impossible. ${ }^{21}$

In the post-analytic phase, screening for a medical condition using urinalysis may lead to unintended consequences based on incidental findings. The perception of abnormal results leads to further action from clinicians either following up with cultures or inappropriately treating with antimicrobials. For example, urinalysis performed in a diabetic patient for proteinuria may incidentally reveal pyuria or bacteriuria, which may trigger unnecessary urine cultures and or inappropriate antibiotic therapy. Patients with proteinuria may have concomitant asymptomatic bacteriuria, but they are not related. ${ }^{41}$ Clinicians, however, will often seize this abnormal result and prematurely mislabel the patient with a diagnosis of UTI. ${ }^{40}$

\section{Optimizing the urinalysis}

Urinalysis and urine dipstick tests are easy and inexpensive screening tests, but their results can have important downstream consequences on urine cultures and antimicrobial prescribing. The level 
Table 1. Urinalysis Components That Can Be Ordered In Lieu of Complete Urinalysis (UA)

\begin{tabular}{lllll}
\hline Component & UA Inflammation & UA Metabolic & UA Renal & Complete UA (For Reference Only) \\
\hline Gross evaluation & & Color, odor, clarity & Color, odor, clarity & Color, odor, clarity \\
\hline Chemical analysis & Leukocyte esterase & $\begin{array}{l}\mathrm{pH} \text {, albumin, specific gravity, } \\
\text { glucose, bilirubin, ketones }\end{array}$ & $\begin{array}{l}\mathrm{pH} \text {, heme, albumin, specific } \\
\text { gravity, glucose, bilirubin }\end{array}$ & $\begin{array}{l}\text { pH, heme, leukocyte esterase, nitrite, albumin, } \\
\text { specific gravity, glucose, bilirubin, ketones }\end{array}$ \\
\hline Microscopic & White blood cells & & $\begin{array}{l}\text { White and red blood cells, } \\
\text { casts, crystals }\end{array}$ & $\begin{array}{l}\text { White and red blood cells, bacteria, yeast, } \\
\text { epithelial cells, casts, crystals }\end{array}$ \\
& & &
\end{tabular}

of pyuria on urinalysis correlates with increasing use of urine cultures and inappropriate antimicrobial prescribing. ${ }^{1}$ Gupta et $\mathrm{al}^{1}$ found that patients who were prescribed antimicrobial therapy for asymptomatic pyuria were not only unlikely to experience any reduction in risk of UTIs or surgical site infections, but also were more likely to develop adverse events like Clostridioides difficile infections. Due to its limited diagnostic utility, the Infectious Disease Society of America guidelines specifically recommend against using pyuria or bacteriuria as a criterion for the diagnosis of UTI or for administering antimicrobial therapy. ${ }^{42}$ Similarly, urinalysis results are not included in the National Healthcare Safety Network definitions of symptomatic UTI. ${ }^{43}$ Hence, diagnostic stewardship interventions should address precursor tests like urinalysis by uncoupling it from urine cultures, interpreting urinalysis results in the context of their pretest probability, and ideally, deconstructing urinalysis into components.

First, a concerted effort should be made to ensure that the urinalysis is only used when it provides significant value to manage a disease, regardless of whether it is an infectious or non-infectious condition. Routine urinalysis screening is a surprisingly common practice, used in $25 \%$ of emergency department visits, but does not directly impact decisions of care and delays the final disposition in most patients. ${ }^{44,45}$ Even though routine urinalysis testing (ie, screening) is presumed to help detect urinary tract malignancy, renal disease, and diabetes, these diseases are rare in young asymptomatic persons, making false-positive and incidental findings more likely. An annual urinalysis is not warranted for screening healthy asymptomatic individuals without major risk factors for bladder cancer (eg, persons with heavy exposure to cigarette smoke and other bladder carcinogens). ${ }^{46,47}$ Similarly, urinalysis has low utility in asymptomatic patients undergoing orthopedic, vascular, or cardiac surgeries. ${ }^{29}$ Hence, urinalysis should not be incorporated in general medical or surgical order sets unless it directly relates to the condition being managed.

Second, the value of urinalysis varies based on the patient characteristics and clinical scenario: catheterized versus noncatheterized patients, symptomatic versus asymptomatic patients, or older versus younger patients. For example, reflex urine cultures are useful when directed toward symptomatic noncatheterized patients, especially in the outpatient and emergency room settings. However, reflex urine cultures have poor utility in catheterized patients, neonates, and neutropenic patients. ${ }^{40}$ Clinicians should use the clinical context to develop a pretest probability for a likely diagnosis to which the urinalysis parameters should be applied. This process will allow the clinician to develop a differential diagnosis using the urinalysis parameters of significance while giving less weight to findings that are likely unrelated to the underlying kidney disease.

Third, we propose a novel diagnostic stewardship approach to consider various elements of urinalysis separately, based on the function they serve. Establishing panels based on common clinical indications for urine testing will allow clinicians to choose the panel that best aligns with their intended use and reduce unnecessary (off-target) testing. Currently, some hospitals limit the use of microscopy in patients that meet specific criteria on urinalysis, while other laboratories limit urine cultures to patients that meet specific urinalysis criteria. ${ }^{27,48}$ Laboratories can consider changing the ordering or reporting of urinalysis parameters to reflect underlying disease states based on the clinician's evaluation: inflammation, metabolic disorders, renal disorders, etc (Table 1). Our suggested panels may serve as a starting point for streamlined evaluation of urine. These panels will require creating distinct orderables and reporting specific parameters based on pretest probability of disease. Making changes to urinalysis at the analytic stage may require manufacturing changes and US Food and Drug Adminstration reviews. An alternative intervention would be suppressing urinalysis components at the post-analytic phase. We encourage laboratories to evaluate their own data related to urinalysis so that these panels can be further refined based on institution specific ordering practices and needs. Using a directed approach to urinalysis would minimize identifying spurious results that may be detected as part of the bundled test of the urinalysis.

Lastly, for any of these interventions to be successful, they will need to be viewed as meaningful and necessary by adopters. More data are needed to evaluate the implementation of disease-specific urinalysis panels and any associated harms and benefits. ${ }^{1,29,49}$ The first step would be to pilot these directed urinalysis panels in certain settings and to evaluate the impact of these interventions on patient outcomes. Sustainability and long-term success will depend on the adaptive component of the intervention and organizational culture. For success in diagnostic stewardship interventions, both leadership and clinicians need to appreciate the ongoing value in these interventions.

In conclusion, the widespread indiscriminate use of urinalysis, especially as screening tests in emergency departments, clinics, hospitals and nursing homes, has led to serious downstream consequences. Abnormal urinalysis parameters in a patient without urinary symptoms is a powerful stimulus to order a urine culture and start antibiotic treatment, thwarting diagnostic and antibiotic stewardship interventions. A re-evaluation of the utility of the urinalysis and deconstructing the urinalysis to fit the diagnostic needs for patient care are critical first steps in mitigating the unnecessary urine cultures, inappropriate antibiotic use, and potential harms.

Acknowledgments. We thank Dr Kenneth Schmader, Professor of Medicine, Director of the GRECC and ACOS for Geriatrics and Extended Care, Duke University, for review of and suggestions for this manuscript.

Financial support. S.A. is supported by NIH-NIDDK K12DK100024 (KURe) for this work.

Conflicts of interest. S.A. reports grants from the CDC, NIH-NIDDK, SHEA, and consulting fees from IPEC Experts, LLC, (unrelated to this work) 


\section{References}

1. Gupta K, O’Brien W, Gallegos-Salazar J, Strymish J, Branch-Elliman W. How testing drives treatment in asymptomatic patients: level of pyuria directly predicts probability of antimicrobial prescribing. Clin Infect Dis 2020;71:614-621.

2. Juthani-Mehta M, Tinetti M, Perrelli E, Towle V, Van Ness PH, Quagliarello V. Diagnostic accuracy of criteria for urinary tract infection in a cohort of nursing home residents. J Am Geriatr Soc 2007;55:1072-1077.

3. Tambyah PA, Maki DG. The relationship between pyuria and infection in patients with indwelling urinary catheters: a prospective study of 761 patients. Arch Intern Med 2000;160:673-677.

4. American Board of Internal Medicine. Choosing Wisely campaigntreatment of asymptomatic bacteriuria. Choosing Wisely website. http:// www.choosingwisely.org/clinician-lists/infectious-diseases-society-antibioticsfor-bacteruria/. Published 2015. Accessed July 15, 2017.

5. Nicolle LE, Gupta K, Bradley SF, et al. Clinical practice guideline for the management of asymptomatic bacteriuria: 2019 update by the Infectious Diseases Society of America. Clin Infect Dis 2019;68:1611-1615.

6. Sullivan KV, Morgan DJ, Leekha S. Use of diagnostic stewardship practices to improve urine culturing among SHEA Research Network hospitals. Infect Control Hosp Epidemiol 2019;40:228-231.

7. Mullin KM, Kovacs CS, Fatica C, et al. A multifaceted approach to reduction of catheter-associated urinary tract infections in the intensive care unit with an emphasis on "stewardship of culturing." Infect Control Hosp Epidemiol 2017;38:186-188.

8. Morgan DJ, Malani P, Diekema DJ. Diagnostic stewardship-leveraging the laboratory to improve antimicrobial use. JAMA 2017;318:607-608.

9. Sarg M, Waldrop GE, Beier MA, et al. Impact of changes in urine culture ordering practice on antimicrobial utilization in intensive care units at an academic medical center. Infect Control Hosp Epidemiol 2016;37:448-454.

10. Waters TM, Daniels MJ, Bazzoli GJ, et al. Effect of Medicare's nonpayment for hospital-acquired conditions: lessons for future policy. JAMA Intern Med 2015;175:347-354.

11. Viehman JA, Saver JL, Liebeskind DS, et al. Utility of urinalysis in discriminating cardioembolic stroke mechanism. Arch Neurol 2007;64:667-670.

12. Cavanaugh C, Perazella MA. Urine sediment examination in the diagnosis and management of kidney disease: core curriculum 2019. Am J Kidney Dis 2019;73:258-272.

13. Cameron JS. A history of urine microscopy. Clin Chem Lab Med 2015;53 suppl 2:s1453-s1464.

14. Echeverry G, Hortin GL, Rai AJ. Introduction to urinalysis: historical perspectives and clinical application. Methods Mol Biol 2010;641:1-12.

15. Yin P, Kiss A, Leis JA. Urinalysis orders among patients admitted to the general medicine service. JAMA Intern Med 2015;175:1711-1713.

16. Rajamanickam A, Noor S, Usmani A. Should an asymptomatic patient with an abnormal urinalysis (bacteriuria or pyuria) be treated with antibiotics prior to major joint replacement surgery? Cleve Clin J Med 2007;74 suppl 1:S17-S18.

17. Pallin DJ, Ronan C, Montazeri K, et al. Urinalysis in acute care of adults: pitfalls in testing and interpreting results. Open Forum Infect Dis 2014; 1(1):ofu019.

18. Bhavsar T, Potula R, Jin M, Truant AL. Predictability of urinalysis parameters in the diagnosis of urinary tract infection: a case study. MLO Med Lab Obs 2015;47:8-13.

19. Van Nostrand JD, Junkins AD, Bartholdi RK. Poor predictive ability of urinalysis and microscopic examination to detect urinary tract infection. Am J Clin Pathol 2000;113:709-713.

20. Abraham G, Reddy YN, George G. Diagnosis of acute pyelonephritis with recent trends in management. Nephrol Dial Transplant 2012;27: 3391-3394.

21. Humphries RM, Dien Bard J. Point-counterpoint: reflex cultures reduce laboratory workload and improve antimicrobial stewardship in patients suspected of having urinary tract infections. J Clin Microbiol 2016;54: 254-258.

22. Khawcharoenporn T, Vasoo S, Ward E, Singh K. Abnormal urinalysis finding triggered antibiotic prescription for asymptomatic bacteriuria in the ED. Am J Emerg Med 2011;29:828-830.
23. Grossfeld GD, Litwin MS, Wolf JS, et al. Evaluation of asymptomatic microscopic hematuria in adults: the American Urological Association best practice policy - part I: definition, detection, prevalence, and etiology. Urology 2001;57:599-603.

24. Grossfeld GD, Wolf JS Jr, Litwan MS, et al. Asymptomatic microscopic hematuria in adults: summary of the AUA best practice policy recommendations. Am Fam Physician 2001;63:1145-1154.

25. Mohr NM, Harland KK, Crabb V, et al. Urinary squamous epithelial cells do not accurately predict urine culture contamination, but may predict urinalysis performance in predicting bacteriuria. Acad Emerg Med 2016;23: 323-330.

26. Walter FG, Gibly RL, Knopp RK, Roe DJ. Squamous cells as predictors of bacterial contamination in urine samples. Ann Emerg Med 1998;31: $455-458$.

27. Foudraine DE, Bauer MP, Russcher A, et al. Use of automated urine microscopy analysis in clinical diagnosis of urinary tract infection: defining an optimal diagnostic score in an academic medical center population. JClin Microbiol 2018;56(6):e02030-17.

28. Kauffman CA. Candiduria. Clin Infect Dis 2005;41 suppl 6:S371-S376.

29. Gallegos Salazar J, O’Brien W, Strymish JM, Itani K, Branch-Elliman W, Gupta K. Association of screening and treatment for preoperative asymptomatic bacteriuria with postoperative outcomes among US veterans. JAMA Surg 2019;154:241-248.

30. Lamb MJ, Baillie L, Pajak D, et al. Elimination of screening urine cultures prior to elective joint arthroplasty. Clin Infect Dis 2017;64:806-809.

31. Sox CM, Christakis DA. Pediatricians' screening urinalysis practices. J Pediatr 2005;147:362-365.

32. Anfinson TJ, Kathol RG. Screening laboratory evaluation in psychiatric patients: a review. Gen Hosp Psychiatry 1992;14:248--257.

33. Horstman MJ, Spiegelman A, Naik AD, Trautner BW. National patterns of urine testing during inpatient admission. Clin Infect Dis 2017;65: 1199-1205.

34. Phillips C. The tyranny of testing for urinary tract infection. Caring for the Ages website. https://www.morx.com/assets/docs/CPEHandouts/caring\% 20for\%20the\%20ages.pdf. Published 2013. Accessed Jan 21, 2021.

35. David TS, Vrahas MS. Perioperative lower urinary tract infections and deep sepsis in patients undergoing total joint arthroplasty. J Am Acad Orthop Surg 2000;8:66-74.

36. Ling D, Seidelman J, Dodds-Ashley E, et al. Navigating reflex urine culture practices in community hospitals: need for a validated approach. Am J Infect Control 2020;48:1549-1551.

37. Munigala S, Rojek R, Wood $\mathrm{H}$, et al. Effect of changing urine testing orderables and clinician order sets on inpatient urine culture testing: analysis from a large academic medical center. Infect Control Hosp Epidemiol 2019; 40:281-286.

38. Hospital-Acquired Condition Reduction Program (HACRP). Centers for Medicare and Medicaid Services website. https://www.cms.gov/Medicare/ Medicare-Fee-for-Service-Payment/AcuteInpatientPPS/HAC-ReductionProgram.html. Published 2018. Updated 07/30/2018. Accessed August 1, 2018.

39. Balas EA, Chapman WW. Road map for diffusion of innovation in health care. Health Aff (Millwood) 2018;37:198-204.

40. Fakih MG, Advani SD, Vaughn VM. Diagnosis of urinary tract infections: need for a reflective rather than reflexive approach. Infect Control Hosp Epidemiol 2019;40:834-835.

41. Carter JL, Tomson CR, Stevens PE, Lamb EJ. Does urinary tract infection cause proteinuria or microalbuminuria? A systematic review. Nephrol Dial Transplant 2006;21:3031-3037.

42. Hooton TM, Bradley SF, Cardenas DD, et al. Diagnosis, prevention, and treatment of catheter-associated urinary tract infection in adults: 2009 International Clinical Practice Guidelines from the Infectious Diseases Society of America. Clin Infect Dis 2010;50:625-663.

43. National Healthcare Safety Network (NHSN) Patient Safety Component Manual. Centers for Disease Control and Prevention website. https:// www.cdc.gov/nhsn/pdfs/pscmanual/pcsmanual_current.pdf. Published January 2017. Accessed January 1, 2017.

44. Anand A, Ballinger B, Ganti L. Impact of urinalysis on medical decisionmaking and length of stay. Cureus 2018;10(4):e2531. 
45. Neyman G, Dalsey W. A Quantification of the impact of awaiting results of a urinalysis upon emergency department length of stay. J Emerg Med 2021; 60:158-164.

46. Akin BV, Hubbell FA, Frye EB, Rucker L, Friis R. Efficacy of the routine admission urinalysis. Am J Med 1987;82:719-722.

47. Kiel DP, Moskowitz MA. The urinalysis: a critical appraisal. Med Clin North Am 1987;71:607-624.
48. Munigala S, Jackups RR Jr, Poirier RF, et al. Impact of order set design on urine culturing practices at an academic medical centre emergency department. BMJ Qual Saf 2018;27:587-592.

49. Zalmanovici Trestioreanu A, Lador A, Sauerbrun-Cutler MT, Leibovici L. Antibiotics for asymptomatic bacteriuria. Cochrane Database Syst Rev. 2015;4:CD009534. 